\title{
MALDI-TOF MS Analysis of Proanthocyanidins in Two Lowland Tropical Forest Species of Cecropia: A First Look at Their Chemical Structures
}

\author{
Alex Van Huynh * and John M. Bevington \\ Department of Biological Sciences, Moravian College, 1200 Main Street, Bethlehem, PA 18018-6650, \\ USA; E-Mail: jmb@cs.moravian.edu \\ * Author to whom correspondence should be addressed; E-Mail: stavh02@moravian.edu; \\ Tel.: +41-44-632-3920.
}

Received: 23 June 2014; in revised form: 5 August 2014 / Accepted: 1 September 2014 /

Published: 12 September 2014

\begin{abstract}
The structural chemistry of proanthocyanidin molecules has been investigated in temperate zone plants, but few studies have been done with plants of the Amazonian lowland tropical wet forests where herbivore pressure is more extensive and diverse. Using MALDI-TOF mass spectrometry, we report unique properties of the proanthocyanidin structural chemistry in two neotropical Cecropia species, C. polystachya, a myrmecophyte with mutualistic ants, and C. sciadophylla, a non-myrmecophyte lacking mutualistic ants. Our preliminary data suggests the presence of reportedly uncommon propelargonidin subunits in a majority of proanthocyanidin oligomers. The presence of 3-O-gallate proanthocyanidin monomers was also detected in the mass spectra of both species. Unlike other studies that have examined species growing at higher latitudes, oligomers composed of procyanidin, propelargonidin, and their 3-O-gallates were present in both Cecropia species while the presence of oligomers containing prodelphinidin units were absent or at lower levels. These distinctive features may suggest that proanthocyanidins in some tropical plant species may be an untapped source of proanthocyanidin structural complexity that warrants further investigation. Several differences between spectra of the two Cecropia species could also point to the presence of anti-herbivore defense tradeoffs between chemical defense quality and biotic defense between the two species.
\end{abstract}

Keywords: proanthocyanidins; condensed tannins; Cecropia sciadophylla; Cecropia polystachya; MALDI-TOF mass spectrometry; anti-herbivore defense tradeoffs 


\section{Introduction}

Proanthocyanidins, also known as condensed tannins, are natural polyphenolic plant products that seem to be produced in most vascular plants through a chloroplast-derived organelle recently described as the "tannosome" [1]. A principal role of these compounds is to act as deterrents to herbivory, probably functioning through protein binding via hydrogen and/or covalent bonding and through the production of oxygen radicals [2-5]. Proanthocyanidins are comprised of flavan-3-ol monomers, most notably catechin and epicatechin, commonly connected through C4-C8 bonds [6,7]. Proanthocyanidin structure has a large degree of potential variation such as hydroxyl substitution on the B ring, stereochemistry of the $\mathrm{C}$ ring, galloylation or glycosylation of the $\mathrm{C}$ ring, monomeric bonding patterns, and degree of polymerization (Figure 1). The unique composition of a given proanthocyanidin structure has been implicated in influencing its biological activity $[8,9]$.

Figure 1. Proanthocyanidin polymer structure and monomeric flavan-3-ol precursors.

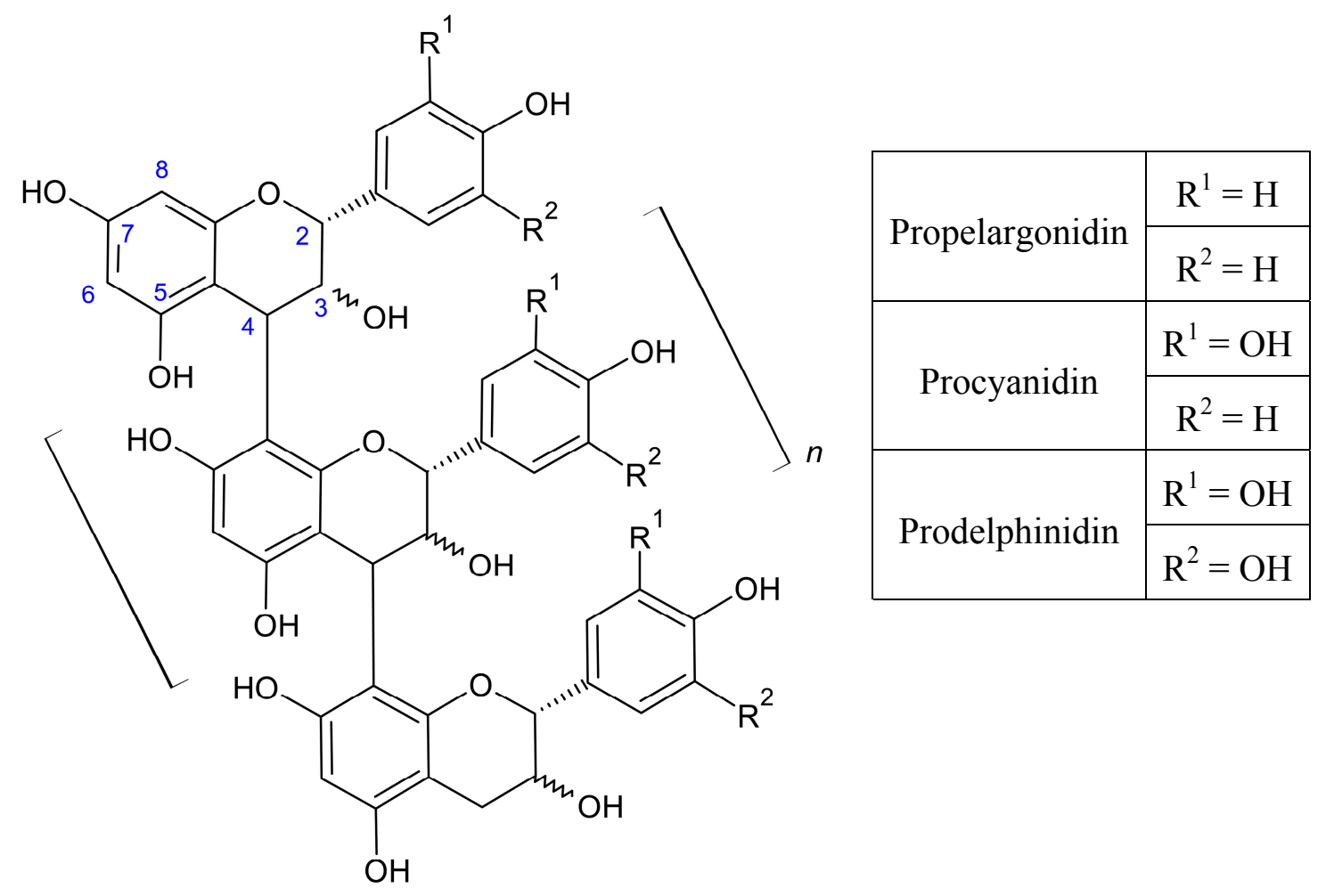

While proanthocyanidin structures and their effects on herbivores have been investigated primarily in temperate zone plants [8], with Quercus spp. being a notable example [10-13], proanthocyanidins in lowland wet forests of the Amazon where tree species diversity and herbivory are hyperdiverse have not been studied extensively [14].

Here we report the first investigation of proanthocyanidin structural chemistry in two lowland tropical species of Cecropia, a neotropical genus of 61 recognized tree species that occupy disturbed areas along riverbanks or in interior tree-fall gaps [15]. Most species are myrmecophytes that develop mutualistic relationships with ants, most notably Azteca species. The ants establish colonies in hollow internodes of the trees and defend the trees against herbivores. The trees in turn provide food for the ants in the form of glycogen-rich Müllerian bodies [15]. Some Cecropia species, however, are 
non-myrmecophytes and do not have ants [15]. In this study, we examined proanthocyanidin structures in C. polystachya, a myrmecophyte, and C. sciadophylla, a non-myrmecophyte.

Because proanthocyanidins may be an important component of chemical defense against herbivores in longer lived trees [16], we hypothesized that any differences in herbivore defense in these two species of Cecropia trees may manifest themselves not only in the presence or absence of mutualistic ants, but also in the structure of their proanthocyanidins. For this reason we used matrix-assisted laser desorption/ionization time of flight mass spectrometry (MALDI-TOF MS) and two dimensional thin layer chromatography (TLC) to examine the structural chemistry of proanthocyanidins of both mature and immature leaves of C. sciadophylla and C. polystachya.

\section{Results and Discussion}

Resolution of proanthocyanidins by MALDI-TOF MS was comparable to the quality of spectra produced in other studies [17-19], supporting the reliability of the extraction procedure in its degree of purification. The characteristic feature marking the presence of proanthocyanidins is an arithmetic series of peaks, each peak $288 \mathrm{Da}$, the mass of an additional procyanidin unit (Figure 1), greater than the previous peak. Thus, each group of peaks represents an oligomer of one greater degree of polymerization than that of the previous peak 288 Da lighter. Proanthocyanidins from immature and mature leaves of $C$. sciadophylla were resolved up to an octamer (Figure 2A,B). Immature leaves of C. polystachya were resolved up to a decamer (Figure 2C). In contrast, the mature leaves of C. polystachya were only resolved up to a tetramer (Figure 2D). The peak of each oligomer of a specific degree of polymerization is further divided into a series of smaller sub-peaks (Figure 3).

Figure 2. MALDI spectrum of proanthocyanidins extracted from Cecropia leaves. The distance between subsequent groups of peaks is $288 \mathrm{Da}$, the mass of an additional procyanidin unit. (A) Cecropia sciadophylla immature leaves $\left(\mathrm{Na}^{+}\right)$. (B) Cecropia sciadophylla mature leaves $\left(\mathrm{Na}^{+}\right)$. (C) Cecropia polystachya immature leaves $\left(\mathrm{Na}^{+}\right)$. (D) Cecropia polystachya mature leaves $\left(\mathrm{Cs}^{+}\right)$.

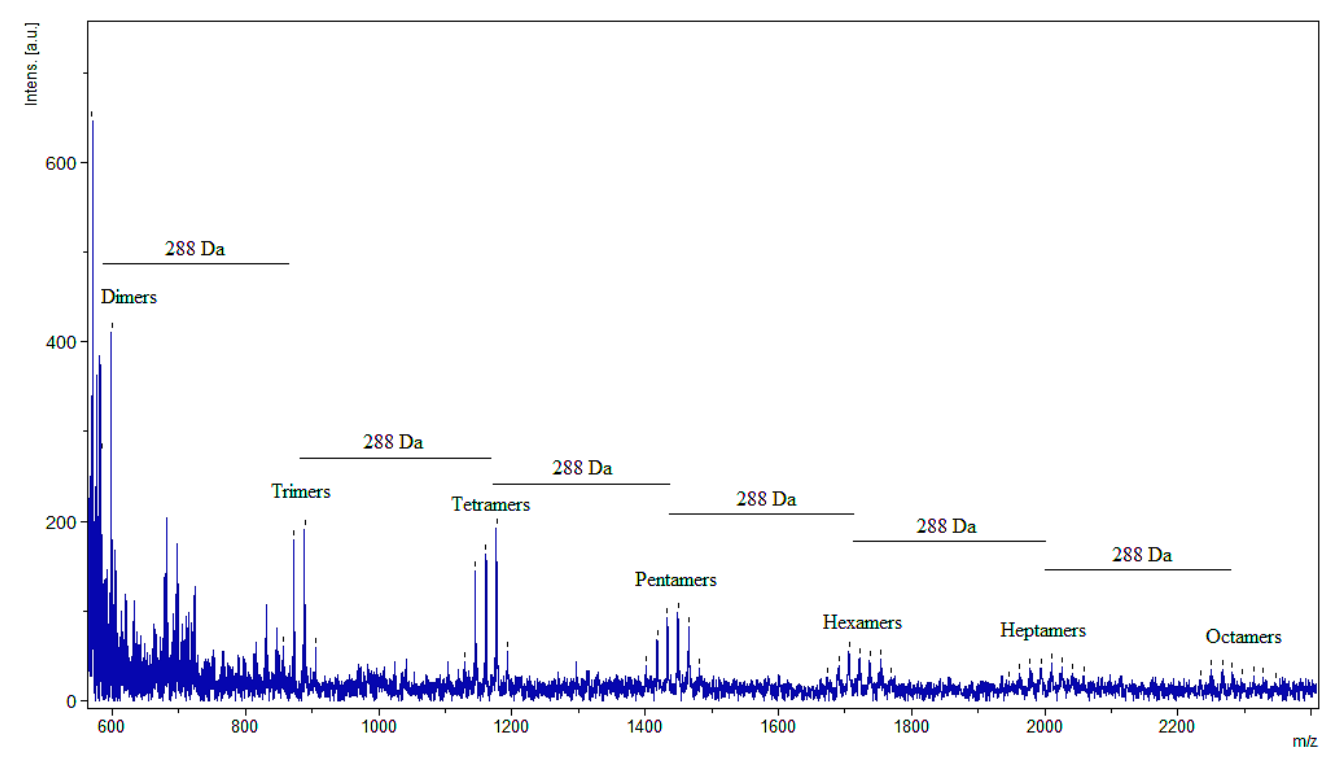

(A) 
Figure 2. Cont.

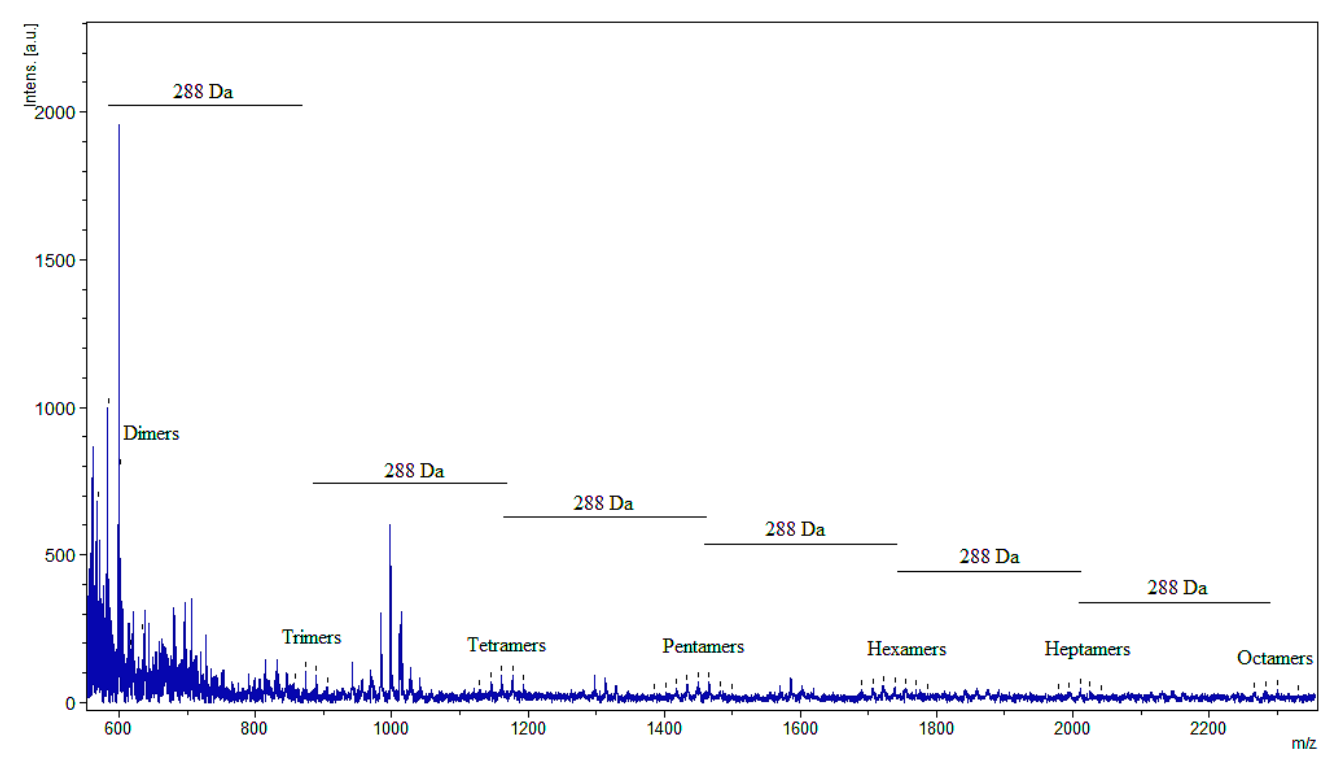

(B)

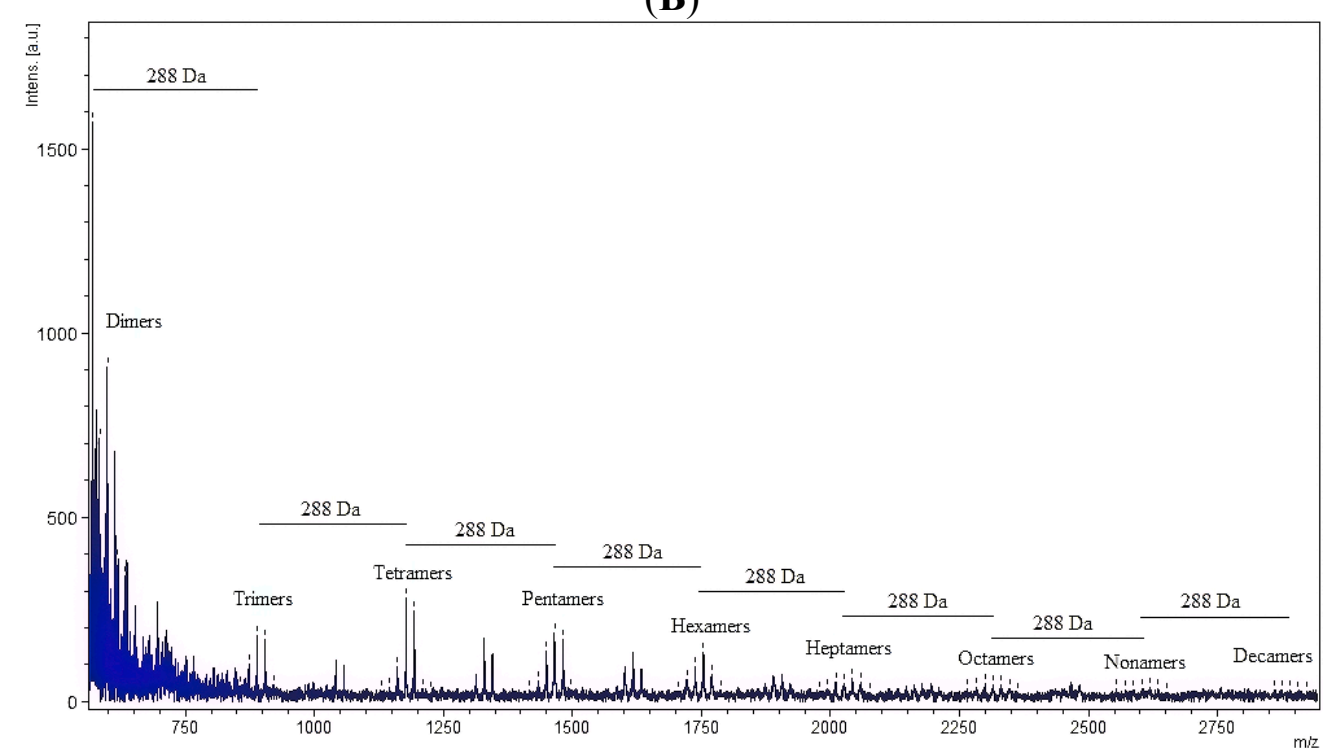

(C)

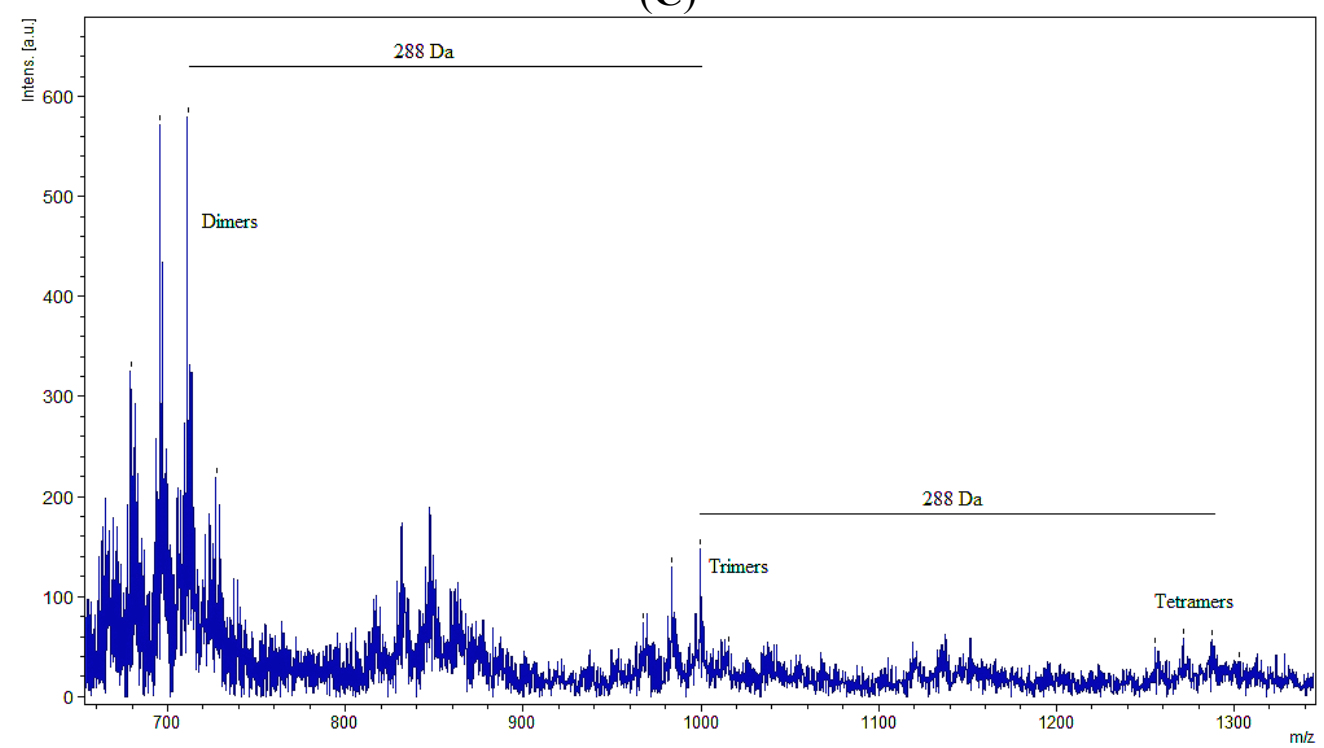

(D) 
Figure 3. Zoomed image illustrating degree of substitution distribution patterns of Cecropia sciadophylla proanthocyanidins extracted from immature leaves. The distance between subsequent peaks is $16 \mathrm{Da}$, the mass of an additional hydroxyl group. PC: procyanidin, PD: prodelphinidin, PP: propelargonidin.

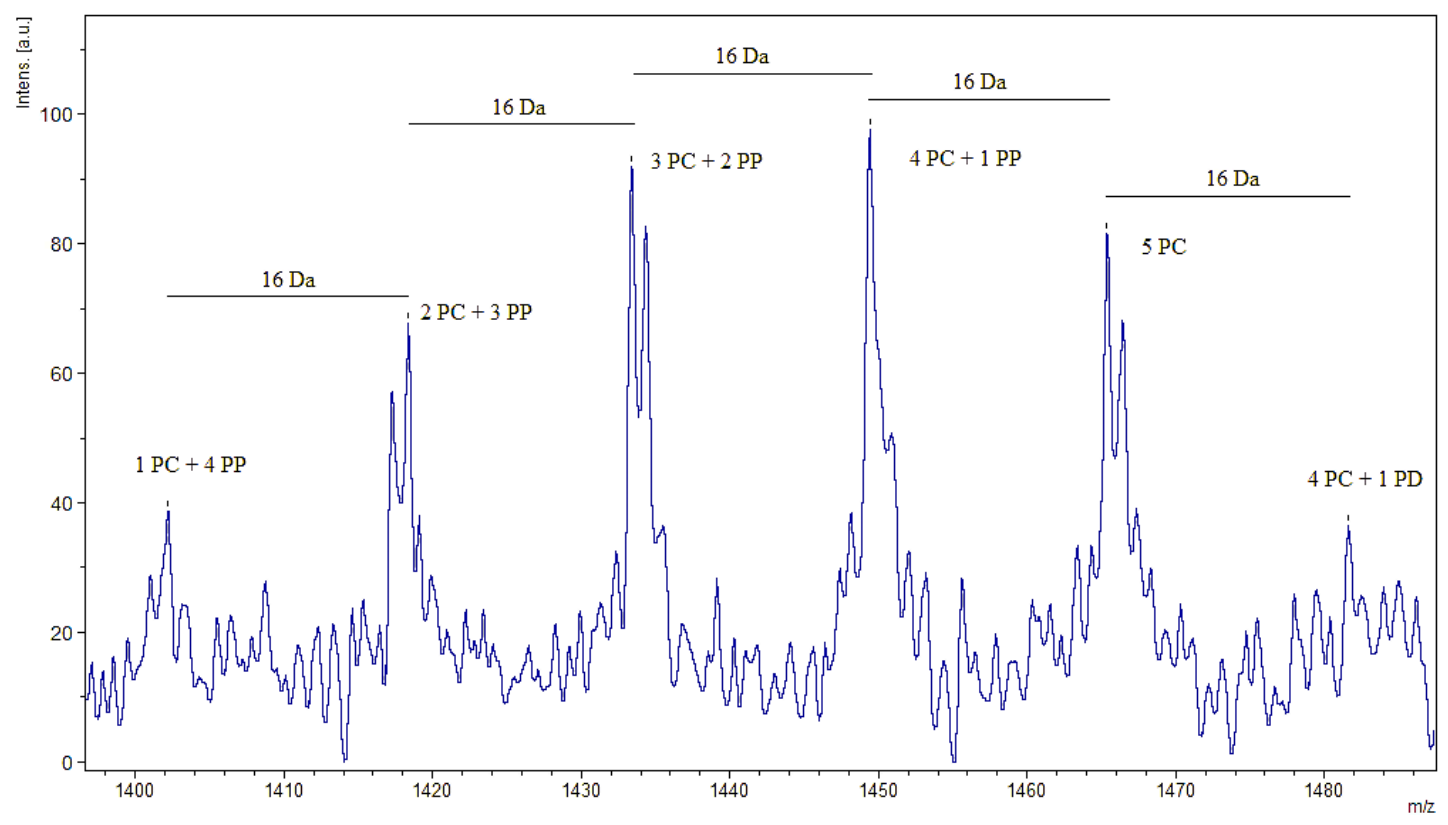

This phenomenon can offer insights into the composition of the types of oligomers present. This is based on the fact that the different potential monomers (procyanidins, prodelphinidins, and propelargonidins) all differ by the number of hydroxyl groups they possess on the B ring. Thus, after calculating the theoretical mass number for a certain sized oligomer consisting entirely of procyanidins, the sub-peaks one finds that are multiples of $16 \mathrm{Da}$ apart (the mass of an added/removed hydroxyl) correspond to the replacement of a procyanidin unit with either a prodelphinidin unit (the addition of a hydroxyl) or with a propelargonidin unit (the removal of a hydroxyl).

From the series of split sub-peaks $16 \mathrm{Da}$ apart that are present for a certain oligomer, the monomeric composition can be inferred using the equation $288 a+304 b+272 c+2+d$ where $a$ is the number of procyanidin units, $b$ is the number of prodelphinidin units, $c$ is the number of propelargonidin units, 2 corresponds to the number of extra hydrogens on the terminal subunits, and $d$ is the atomic mass of the ionizing agent (e.g., $\mathrm{Na}^{+}$). Using this analysis, the monomeric composition of the oligomer representative of each sub-peak can be calculated in the form of the number of each type of monomeric component (Table 1). Table 1 is representative of the remaining three samples.

Sub-splitting analysis revealed that the majority of peaks for all four samples represent oligomers contain procyanidin and propelargonidin units. Peaks representing the presence of prodelphinidin units for given oligomers in each of the four samples were in the minority if not absent altogether. The possibility of potassium as the ionizing agent is a potential confounding factor and this fact may account for the observation of prodelphinidin containing molecules since the replacement of $\mathrm{Na}^{+}$with $\mathrm{K}^{+}$as the ionizing agent would result in a 16 Da mass increase. 
Table 1. Observed and calculated masses for Cecropia sciadophylla proanthocyanidins extracted from immature leaves. Mass calculations were based on the equation $288 a+$ $304 b+272 c+2+23$, where $a$ is the number of procyanidin units, $b$ is the number of prodelphinidin units, and $c$ is the number of propelargonidin units, 2 corresponds to the number of extra hydrogens on the terminal subunits and 23 is the atomic mass of sodium.

\begin{tabular}{|c|c|c|c|c|c|}
\hline $\begin{array}{c}\text { Degrees of } \\
\text { Polymerization }\end{array}$ & $\begin{array}{c}\text { Number of } \\
\text { Procyanidin } \\
\text { Units }(a) \\
\end{array}$ & $\begin{array}{c}\text { Number of } \\
\text { Prodelphinidin } \\
\text { Units }(b)\end{array}$ & $\begin{array}{c}\text { Number of } \\
\text { Propelargonidin } \\
\text { Units (c) } \\
\end{array}$ & $\begin{array}{c}\text { Calculated } \\
{[\mathrm{M}+\mathrm{Na}]^{+}}\end{array}$ & $\begin{array}{c}\text { Observed } \\
{[\mathbf{M}+\mathbf{N a}]^{+}}\end{array}$ \\
\hline \multirow{3}{*}{ Dimer } & 0 & 0 & 2 & 569 & 569 \\
\hline & 1 & 0 & 1 & 585 & 585 \\
\hline & 2 & 0 & 0 & 601 & 601 \\
\hline \multirow{4}{*}{ Trimer } & 1 & 0 & 2 & 857 & 857 \\
\hline & 2 & 0 & 1 & 873 & 873 \\
\hline & 3 & 0 & 0 & 889 & 889 \\
\hline & 2 & 1 & 0 & 905 & 905 \\
\hline \multirow{5}{*}{ Tetramer } & 1 & 0 & 3 & 1129 & 1129 \\
\hline & 2 & 0 & 2 & 1145 & 1145 \\
\hline & 3 & 0 & 1 & 1161 & 1161 \\
\hline & 4 & 0 & 0 & 1177 & 1177 \\
\hline & 3 & 1 & 0 & 1193 & 1193 \\
\hline \multirow{6}{*}{ Pentamer } & 1 & 0 & 4 & 1401 & 1402 \\
\hline & 2 & 0 & 3 & 1417 & 1418 \\
\hline & 3 & 0 & 2 & 1433 & 1433 \\
\hline & 4 & 0 & 1 & 1449 & 1449 \\
\hline & 5 & 0 & 0 & 1465 & 1465 \\
\hline & 4 & 1 & 0 & 1481 & 1482 \\
\hline \multirow{7}{*}{ Hexamer } & 1 & 0 & 5 & 1673 & 1674 \\
\hline & 2 & 0 & 4 & 1689 & 1691 \\
\hline & 3 & 0 & 3 & 1705 & 1706 \\
\hline & 4 & 0 & 2 & 1721 & 1722 \\
\hline & 5 & 0 & 1 & 1737 & 1738 \\
\hline & 6 & 0 & 0 & 1753 & 1754 \\
\hline & 5 & 1 & 0 & 1769 & 1770 \\
\hline \multirow{8}{*}{ Heptamer } & 1 & 0 & 6 & 1945 & 1947 \\
\hline & 2 & 0 & 5 & 1961 & 1962 \\
\hline & 3 & 0 & 4 & 1977 & 1978 \\
\hline & 4 & 0 & 3 & 1993 & 1994 \\
\hline & 5 & 0 & 2 & 2009 & 2010 \\
\hline & 6 & 0 & 1 & 2025 & 2026 \\
\hline & 7 & 0 & 0 & 2041 & 2042 \\
\hline & 6 & 1 & 0 & 2057 & 2059 \\
\hline \multirow{8}{*}{ Octamer } & 2 & 0 & 6 & 2233 & 2233 \\
\hline & 3 & 0 & 5 & 2249 & 2250 \\
\hline & 4 & 0 & 4 & 2265 & 2267 \\
\hline & 5 & 0 & 3 & 2281 & 2281 \\
\hline & 6 & 0 & 2 & 2297 & 2296 \\
\hline & 7 & 0 & 1 & 2313 & 2313 \\
\hline & 8 & 0 & 0 & 2329 & 2329 \\
\hline & 7 & 1 & 0 & 2345 & 2347 \\
\hline
\end{tabular}

These findings were surprising because propelargonidin monomers are rare in nature and are not often seen in plant proanthocyanidins [6,7]. Ayres et al. [8] found no presence of propelargonidin units in 16 woody plant species over six families and Zhang and Lin [19], using MALDI-TOF MS, found 
that proanthocyanidins in Japanese Oak (Lithocarpus glaber) contained only procyanidin and prodelphinidin units. Behrens et al. [17], using MALDI-TOF MS, found that lime (Tilia cordata) and willow (Salix alba) appeared to contain only procyanidin units while spruce (Picea abies) contained mixtures of procyanidin and prodelphinidin units. Behrens et al [17] did find that beech (Fagus sylvatica) appeared to contain minor peaks reflecting the presence of propelargonidin units but these were not as prevalent as those reflecting a combination of procyanidin and prodelphinidin units. Although our data is strongly consistent with the presence of appreciable propelargonidin containing oligomers, without the benefit of HPLC, we propose a tentative assignment of propelargonidin containing proanthocyanidins in these two species of Cecropia.

Spectra for all samples also revealed the presence of oligomers containing at least one 3-O-gallate flavan-3-ol derivative (Figure 4). These subunits containing a galloyl ester off of carbon 3 on the $\mathrm{C}$ ring in place of a normal hydroxyl are visually discernible in the spectra by the presence of an arithmetic series of peaks shifted 152 Da upwards from the original non-3-O-gallate oligomeric series. While the presence of multiple subunits possessing a galloyl ester may be present in a single proanthocyanidin oligomer, the ability to discern the exact number is limited by the MALDI mass spectra. While the presence of one galloyl ester is easily seen via the peak series 152 Da greater than the original non-galloyl ester series, the presence of two galloyl esters would result in a shifted spectrum 304 Da upwards. This potential peak overlaps directly with a variant of the next original non-3-O-gallate containing oligomer. Thus, the presence of two galloyl esters on one oligomer and the next oligomer possessing one greater degree of polymerization mask each other. The peaks designated as representing an oligomer containing a prodelphinidin unit (Figure 3) may be a result of potassium ionization of a non-prodelphinidin containing oligomer or an oligomer containing two galloyl ester substituents.

Figure 4. MALDI spectrum of Cecropia polystachya proanthocyanidins extracted from immature leaves. Displayed is the polymerization pattern for sodium ions with polymers possessing one galloyl ester (marked with ticks). The distance between subsequent groups of peaks is $288 \mathrm{Da}$, the mass of an additional procyanidin unit. The shifted distance from the original peak series is $152 \mathrm{Da}$, the mass of one added galloyl ester substituent.

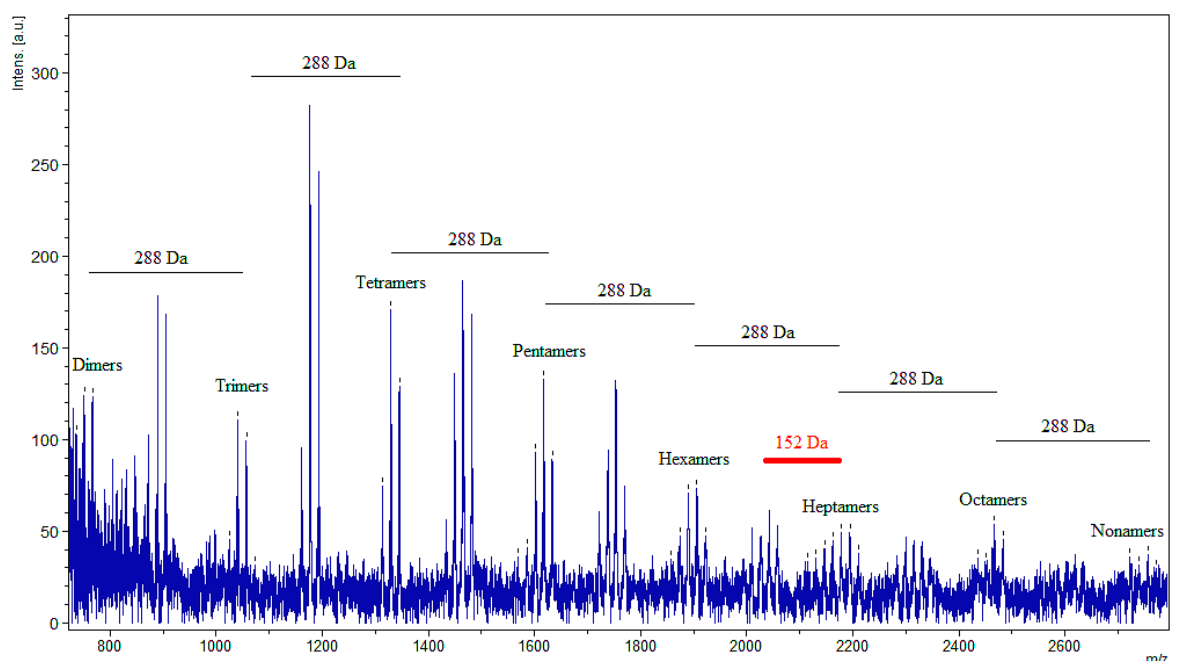

Thiolysis of each of the four samples yielded marked differences between species in the number of vanillin/ $\mathrm{HCl}$ reaction spots. The immature C. sciadophylla leaf yielded six spots (Figure 5A) and the 
mature leaf yielded five spots (Figure 5B). In contrast, the immature $C$. polystachya leaf yielded three spots (Figure 5C) and the mature leaf yielded only two spots (Figure 5D). The heterogeneous proanthocyanidin composition in C. sciadophylla contrasts with temperate zone plants such as blackbrush (Coleogyne ramosissima) and low-brush cranberry (Vacciniumvitis-idaea), both of which possess proanthocyanidins comprised entirely of procyanidin units [8].

Figure 5. Two dimensional TLC of thiolyzed proanthocyanidins extracted from Cecropia leaves. (A) Cecropia sciadophylla immature leaves. (B) Cecropia sciadophylla mature leaves. (C) Cecropia polystachya immature leaves. (D) Cecropia polystachya mature leaves.

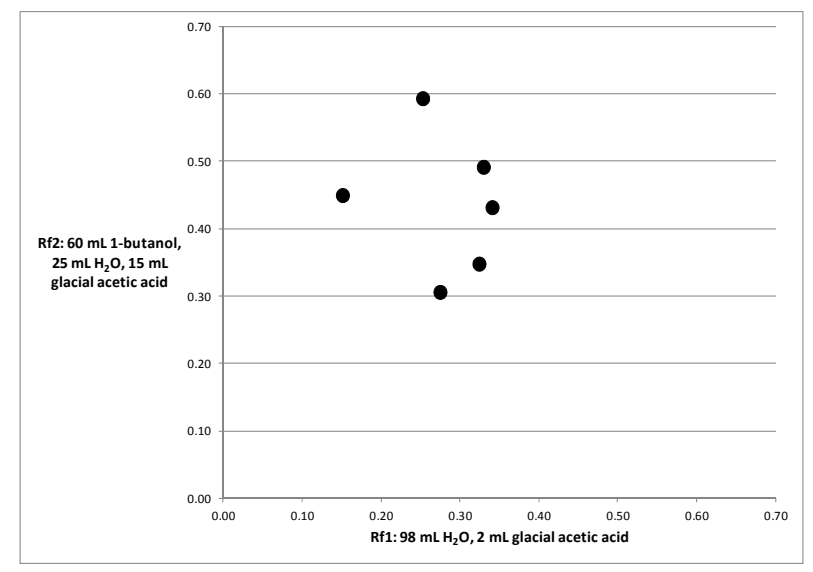

(A)

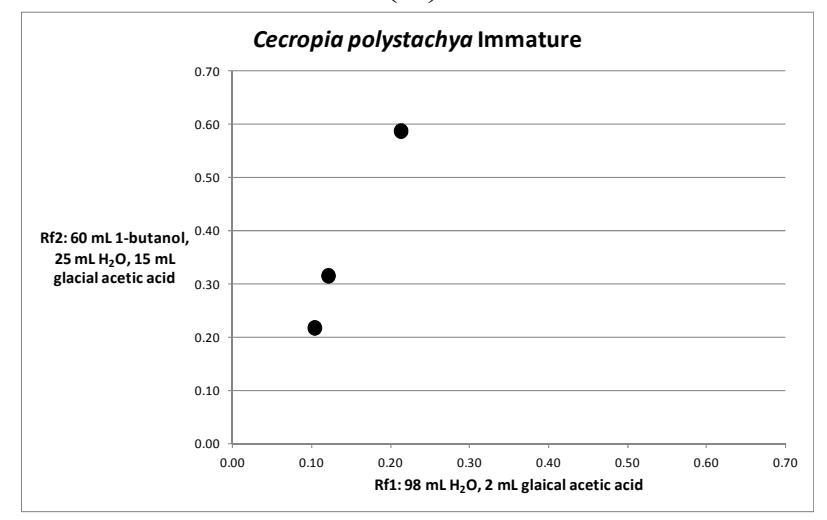

(C)

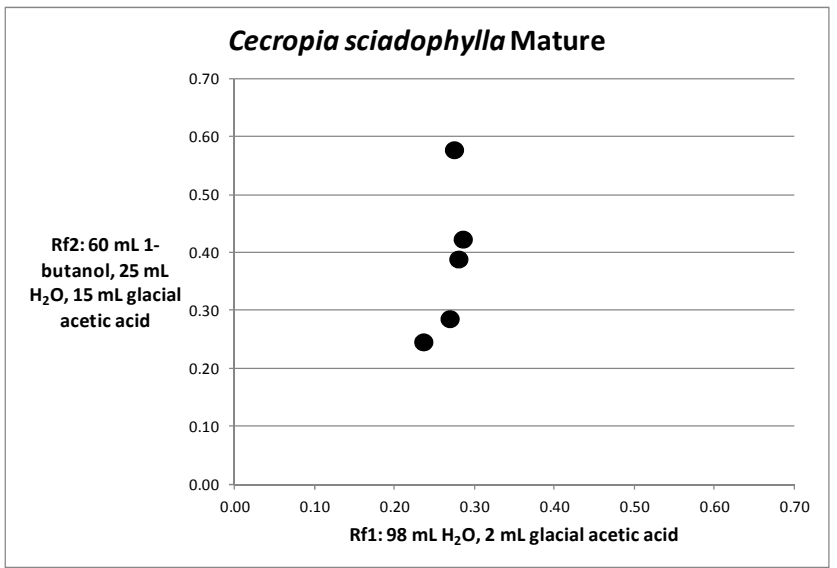

(B)

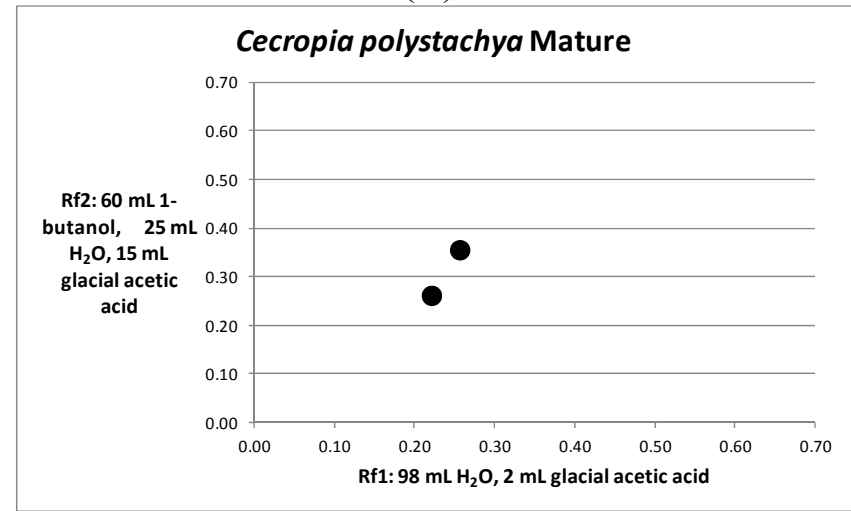

(D)

In comparing our results between the two species, TLC analysis of thiolyzed proanthocyanidins showed that the composition of proanthocyanidin monomers from both the immature and mature leaves of $C$. sciadophylla was more heterogeneous than from the leaves of C. polystachya. This is interesting because it might have ecological significance. $C$. polystachya is defended from herbivores by colonies of aggressive ants. Anti-herbivore defense in C. sciadophylla is related to the chemical and physical properties of its leaves. The greater chemical heterogeneity of the proanthocyanidin monomers in C. sciadophylla raises the possibility that the chemical properties of its proanthocyanidins may contribute to its anti-herbivore defense. We have previously found that the leaves of C. sciadophylla, particularly the immature leaves, contain high levels of chemical defense in the form of proanthocyanidins and protein precipitable phenolics, and that these levels are significantly greater than those found in C. membranacea, another myrmecophyte species [20]. These findings may reflect 
a tradeoff between biotic defense and chemical defense, especially in the immature leaves [21]. Such tradeoffs have been demonstrated with other genera containing ant mutualisms such as Macaranga [22], Inga [23], and Acacia [24]. Further work will be required to determine if this is the case.

\section{Experimental}

\subsection{Sample Collection}

Two Cecropia species, C. sciadophylla and C. polystachya, were collected in premontane forest along the Alto Madre de Dios River in Manu Park, Peru (12³9'170"S, 71¹4'246"W; elevation of $437 \mathrm{~m}$ asl). Trees were approximately $1 \mathrm{~km}$ apart from each other and were chosen based on the presence of both mature and immature leaves. Leaves were harvested, measured, and dried in a plant press. Blotters and driers were changed frequently. Dry leaves were transferred in newspapers to large, air-tight Ziploc ${ }^{\circledR}$ bags with silica gel contained in paper pouches.

\subsection{Proanthocyanidin Extraction}

Proanthocyanidin extraction procedures were adapted from extraction steps found in several studies examining plant proanthocyanidin structure [16,18,25-27]. Tissue samples (1 gm) of dry leaf tissue were cut into small strips, soaked overnight at $4{ }^{\circ} \mathrm{C}$ in $70 \%$ aqueous acetone, ground in chilled mortars, and extracted three times with cold $70 \%$ aqueous acetone $(25 \mathrm{~mL})$. The combined acetone extracts were subjected to rotary evaporation under reduced pressure at $35-40{ }^{\circ} \mathrm{C}$ to remove the acetone. Aqueous samples were then extracted twice in a separatory funnel with equal volumes of hexanes to remove lipophilic substances, ethyl acetate to remove chlorophyll, and chloroform to remove carotenoids and monomeric phenols. Each aqueous sample was again subjected to rotary evaporation under the same conditions to remove any residual organic solvent. The samples were then filtered through Number 43 Whatman filter paper. The samples were run through a Sephadex LH-20 liquid chromatography column. Carbohydrates and hydrolyzable tannins were eluted with $80 \%$ aqueous ethanol while proanthocyanidins adhered to the column. Proanthocyanidins were later eluted using $70 \%$ acetone. A final rotary evaporation resulted in aqueous proanthocyanidin extracts which were stored at $4{ }^{\circ} \mathrm{C}$ in plastic screw cap vials.

\subsection{MADLI-TOF Mass Spectrometry}

From reports of success with obtaining MALDI-TOF mass spectra of proanthocyanidins isolated from plant material, 2,5-dihydroxybenzoic acid (DHB, Aldrich, St. Louis, MO, USA) was selected as a matrix to crystallize the proanthocyanidin samples [17-19]. Ten $\mathrm{mg}$ of DHB was dissolved in $1 \mathrm{~mL}$ of deionized water and was mixed with the aqueous proanthocyanidin extracts from each species at a 3:1 volumetric ratio. A volume of $2 \mu \mathrm{L}$ of each analyte-matrix solution was applied to a stainless steel target and allowed to dry and crystalize for 10-15 minutes before being subjected to irradiation and ionization with either sodium or cesium ions [28]. Samples were pulsed for 130 ns with a nitrogen laser $(\lambda=337 \mathrm{~nm})$ on a Bruker Daltonics Autoflex instrument (Bruker, Billerica, MA, USA). Spectra were taken in positive deflection mode with an accelerating voltage of $19.0 \mathrm{kV}$ and a reflector voltage of $20.0 \mathrm{kV}$. Spectra were obtained from 100-150 shots and calibrated using ACTH 18-39 (2465.20 Da), angiotensin II (1046.54 Da), bradykinin 1-7 (757.40 Da), and P14R (1533.86 Da) (Bruker). 


\subsection{Thiolysis and Two Dimensional TLC}

A volume of $200 \mu \mathrm{L}$ of aqueous proanthocyanidin extracts was mixed with $200 \mu \mathrm{L}$ of cysteamine thiolysis mixture $(20 \mu \mathrm{L}$ of concentrated $\mathrm{HCl}, 930 \mu \mathrm{L}$ of methanol, and $50 \mathrm{mg}$ of cysteamine hydrochloride) as described by Torres and Lozano [29]. Thiolysis was carried out in an $80{ }^{\circ} \mathrm{C}$ water bath in sealed water-tight microfuge tubes. The reaction was stopped with $1.2 \mathrm{~mL}$ of $0.1 \%$ aqueous trifluoroacetic acid after 4 hours. A $25 \mu \mathrm{L}$ sample of the thiolysis product, applied in $2 \mu \mathrm{L}$ applications, was analyzed via TLC on $20 \times 20 \mathrm{~cm}$ cellulose plates (Merck KGaA, Darmstadt, Germany). The first dimension solvent was deionized water $(98 \mathrm{~mL})$ and glacial acetic acid $(2 \mathrm{~mL})$. The second dimension solvent was 1-butanol $(60 \mathrm{~mL})$, deionized water $(25 \mathrm{~mL})$, and glacial acetic acid $(15 \mathrm{~mL})$ [30]. Completed plates were sprayed with a vanillin/ $\mathrm{HCl}$ reagent $(1 \mathrm{gm}$ vanillin in $10 \mathrm{~mL}$ of concentrated $\mathrm{HCl}$ ). This spray specifically reacts with proanthocyanidins and their monomeric components to produce $\mathrm{red} / \mathrm{pink}$ spots $[6,29]$.

\section{Conclusions}

Here we report a first look at proanthocyanidin structure in Cecropia and one of the few investigations of proanthocyanidins in tropical plants of the Amazonian lowland wet forests. Our data suggest a unique feature of multiple propelargonidin units in Cecropia proanthocyanidins, the presence of galloylated monomers, and a relatively high level of heterogeneity in proanthocyanidin composition compared to temperate zone plants previously reported. Differences in proanthocyanidin composition between the myrmecophyte and non-myrmecophyte Cecropia species were also observed, specifically in number of monomeric components. These differences are intriguing, as they may reflect tradeoffs in anti-herbivore defense that are a function of the level of biotic defense. Further chemical analysis such as MALDI-TOF MS post source decay analysis, HPLC, and NMR studies can confirm the presence of the suggested propelargonidin units presented here and elucidate additional structural information about these apparently unique proanthocyanidins. Herbivore studies using isolated Cecropia proanthocyanidins would provide information on their effects on herbivore growth and development that may reflect important chemically mediated ecological tradeoffs between the two species.

\section{Acknowledgments}

A.V.H. gratefully acknowledges financial support from the Garden Club of America and a Moravian College Student Opportunities for Academic Research (SOAR) award. We thank the Amazon Conservation Association for use of Wayquecha Biological Station and the Peruvian Ministry of Natural Resources for permission to conduct the study. We would also like to thank Stephen Dunham for technical assistance. Publication cost was partially covered by a Moravian College FDRC award to J.M.B.

\section{Author Contributions}

The concept for this study was a product of both authors as was writing the paper. A.V.H. performed the experiments as part of a senior Honors research project. J.M.B. located and identified the trees, collected leaves, recorded field data, and coordinated logistics and transport in Peru. 


\section{Conflicts of Interest}

The authors declare no conflict of interest.

\section{References}

1. Brillouet, J.; Romieu, C.; Schoefs, B.; Solymosi, K.; Cheynier, V.; Fulerand, H.; Verdeil, J.; Gonejero, G. The tannosome is an organelle forming condensed tannins in the chlorophyllous organs of Tracheophyta. Ann. Bot. 2013, 112, 1003-1014

2. Appel, H.M. The Chewing Herbivore Gut Lumen: Physicochemical Conditions and Their Impact on Plant Nutrients, Allelochemicals, and Insect Pathogens. In Insect-Plant Interactions; Bernays, E.A., Ed.; CRC Press: Boca Raton, FL, USA, 1994; pp. 209-223.

3. Baxter, N.J.; Lilley, T.H.; Haslam, E.; Williamson, M.P. Multiple interactions between polyphenols and a salivary proline-rich protein repeat result in complexation and precipitation. Biochemistry 1997, 36, 5566-5577.

4. Felton, G.W.; Duffey, S.S. Reassessment of the role of gut alkalinity and detergency in insect herbivory. J. Chem. Ecol. 1991, 17, 1821-1836.

5. Lee, K. Glutathione S-transferase activities in phytophagous insects: induction and inhibition by plant phytotoxins and phenols. Insect Biochem. 1991, 21, 353-361.

6. Hagerman, A.E. The Tannin Handbook. Available online: http://www.users.muohio.edu/hagermae/ (accessed on 1 September 2013).

7. Porter, L.J. Tannins. In Methods in Plant Biochemistry; Herborne, J.B., Ed.; Academic Press Inc.: San Diego, CA, USA, 1989; Volume 1, pp. 389-419.

8. Ayres, M.P.; Clausen, T.P.; MacLean, S.F.; Redman, A.M.; Reichardt, P.B. Diversity of structure and antiherbivore activity in condensed tannins. Ecology 1997, 78, 1696-1712.

9. Plumb, G.W.; de Pascual-Teresa, S.; Santos-Buelga, C.; Cheynier, V.; Williamson, G. Antioxidant properties of catechins and proanthocyanidins: Effect of polymerization, galloylation and glycosylation. Free Radic. Res. 1998, 19, 351-358.

10. Feeny, P.P. Effect of oak leaf tannins on larval growth of the winter moth Operophtera brumata. J. Insect Physiol. 1968, 14, 805-817.

11. Feeny, P.P. Inhibitory effect of oak leaf tannins on the hydrolysis of proteins by trypsin. Phytochemistry 1969, 8, 2119-2126.

12. Feeny, P.P. Seasonal changes in oak leaf tannins and nutrients as a cause of spring feeding by winter moth caterpillars. Ecology 1970, 51, 565-581.

13. Forkner, R.E.; Marquis, R.J.; Lill, J.T. Feeny revisited: condensed tannins as anti-herbivore defenses in leaf-chewing herbivore communities of Quercus. Ecol. Entomol. 2004, 29, 174-187.

14. Ter Steege, H.; Pitman, N.C.A.; Sabatier, D.; Baraloto, C.; Salomao, R.P.; Guevara, J.E.; Phillips, O.L.; Castilho, C.V.; Magnusson, W.E.; Molino, J.; et al. Hyperdominance in the Amazonian tree flora. Science 2013, 342, doi:10.1126/science.1243092.

15. Berg, C.C.; Rosselli, P.F. Cecropia; New York Botanical Garden Press: Bronx, NY, USA, 2005.

16. Coley, P.D.; Bryant, J.P.; Chapin, F.S. Resource availability and plant antiherbivore defense. Science 1985, 230, 895-899. 
17. Behrens, A.; Maie, N.; Knicker, H.; Kogel-Knabner, I. MALDI-TOF mass spectrometry and PSD fragmentation as means for the analysis of condensed tannins in plant leaves and needles. Phytochemistry 2003, 62, 1159-1170.

18. Foo, L.Y.; Lu, Y.; Howell, A.B.; Vorsa, N. The structure of cranberry proanthocyanidins which inhibit adherence of uropathogenic P-fimbriated Escherichia coli in vitro. Phytochemistry 2000, 54, 173-181.

19. Zhang, L.L.; Lin, Y.M. HPLC, NMR and MALDI-TOF MS analysis of condensed tannins from Lithocarpus glaber leaves with potent free radical scavenging activity. Molecules 2008, 13, 2986-2997.

20. Latterman, T.A.; Mead, J.E.; DuVall, M.A.; Bunting, C.C.; Bevington, J.M. Differences in anti-herbivore defenses in non-myrmecophyte and myrmecophyte Cecropia trees. Biotropica 2014, in press.

21. Coley, P.D. Herbivory and defensive characteristics of tree species in a lowland tropical forest. Ecol. Monogr. 1983, 53, 209-233.

22. Eck, G.; Fiala, B.; Linsenmair, K.E.; Hashim, R.B.; Proksch, P. Trade-off between chemical and biotic antiherbivore defense in the south east Asian plant genus Macaranga. J. Chem. Ecol. 2001, 27, 1979-1996.

23. Koptur, S. Alternative defenses against herbivores in Inga (Fabaceae: Mimosoidae) over an elevational gradient. Ecology 1985, 66, 1639-1650.

24. Rehr, S.S.; Feeny, P.P.; Janzen, D.H. Chemical defense in Central American non-ant-acacias. J. Anim. Ecol. 1973, 42, 405-416.

25. Jones, W.T.; Broadhurst, R.B.; Lyttleton, J.W. The condensed tannins of pasture legume species. Phytochemistry 1976, 15, 1407-1409.

26. Lin, Y.M.; Liu, J.W.; Xiang, P.; Lin, P.; Ye, G.F.; da Sternberg, L.S.L. Tannin dynamics of propagules and leaves of Kandelia candel and Bruguiera gymnorrhiza in the Jiulong River Estuary, Fujian, China. Biogeochemistry 2006, 78, 343-359.

27. Preston, C.M. Condensed Tannins of Salal (Gaultheria Shallon Pursh): A Contributing Factor to Seedling "Growth Check" on Northern Vancouver Island? In Plant Polyphenols 2: Chemistry, Biology, Pharmacology, Ecology; Gross, G.G., Hemingway, R.W., Yoshida, T., Eds.; Kluwer Academic/Plenum Publishers: New York, NY, USA, 1999; pp. 825-841.

28. Xiang, P.; Lin, Y.; Lin, P.; Xiang, C. Effects of adduct ions on matrix-assisted laser desorption/ionization time of flight mass spectrometry of condensed tannins: A prerequisite knowledge. Chin. J. Anal. Chem. 2006, 34, 1019-1022.

29. Torres, J.L.; Lozano, C. Chromatographic characterization of proanthocyanidins after thiolysis with cysteamine. Chromatographica 2001, 54, 523-526.

30. Makkar, H.P.S. Quantification of Tannins in Tree and Shrub Foliage; Springer: Heidelberg, The Netherlands, 2003; pp. 89-93.

Sample Availability: Leaf samples are available from the authors.

(C) 2014 by the authors; license MDPI, Basel, Switzerland. This article is an open access article distributed under the terms and conditions of the Creative Commons Attribution license (http://creativecommons.org/licenses/by/3.0/). 